\title{
Goma de mascar mentolada no manejo da sede pré-operatória: ensaio clínico randomizado*
}

\author{
Aline Korki Arrabal Garcia $1,2,3$ \\ (1D) https://orcid.org/0000-0001-8648-9887 \\ Rejane Kiyomi Furuya ${ }^{4}$ \\ (D) https://orcid.org/0000-0003-0885-5364 \\ Marilia Ferrari Conchon ${ }^{5}$ \\ (D) https://orcid.org/0000-0003-0393-1559 \\ Edilaine Giovanini Rossetto ${ }^{6}$ \\ (1D) https://orcid.org/0000-0002-0996-5154 \\ Rosana Aparecida Spadoti Dantas 5 \\ (D) https://orcid.org/0000-0002-3050-7000 \\ Ligia Fahl Fonseca ${ }^{6}$ \\ (DD https://orcid.org/0000-0001-7550-9141
}

Objetivo: avaliar a efetividade da goma de mascar mentolada em aliviar a intensidade e o desconforto da sede do paciente cirúrgico no período pré-operatório. Método: ensaio clínico controlado randomizado, com 102 pacientes em período pré-operatório, aleatorizados em grupo-controle, com cuidado usual, e grupo experimental, que recebeu goma de mascar mentolada, a variável de tratamento do estudo. O desfecho clínico primário foi a variação da intensidade da sede, avaliada pela Escala Verbal Numérica, e o secundário, a variação do desconforto da sede, avaliada pela Escala de Desconforto da Sede Perioperatória. Teste de Mann-Whitney foi usado para comparar as medidas entre os grupos. Nível de significância adotado de 0,05. Resultados: a goma de mascar mentolada reduziu significativamente a intensidade $(p<0,001)$, com d de Cohen de efeito médio, e o desconforto da sede $(p<0,001)$, com d de Cohen de efeito grande. Conclusão: a goma de mascar mentolada mostrou-se efetiva na redução da intensidade e do desconforto da sede pré-operatória. A estratégia mostrou-se uma opção inovadora, viável e segura no uso para o paciente cirúrgico, no manejo da sede pré-operatória, em cirurgias eletivas. NCT: 03200197.

Descritores: Sede; Goma de Mascar; Mentol; Período Pré-operatório; Saliva; Mastigação.

\section{Como citar este artigo}

Garcia AKA, Furuya RK, Conchon MF, Rossetto EG, Dantas RAS, Fonseca LF. Menthol chewing gum on preoperative thirst management: randomized clinical trial. Rev. Latino-Am. Enfermagem. 2019;27:e3180. [Access † f f ]; Available in: DOI: http://dx.doi.org/10.1590/1518-8345.3070.3180. 


\section{Introdução}

A sede é um sintoma presente, intenso e estressor no pré-operatório. Neste período, o paciente está sujeito a uma série de desconfortos durante o preparo para o procedimento anestésico-cirúrgico. Emoções como medo, ansiedade e estresse deflagram reações fisiológicas, entre elas, a inibição da produção salivar, causando o ressecamento da cavidade orofaríngea ${ }^{(1)}$. No entanto, esse não é o único desafio que o paciente enfrenta.

No período pré-operatório, à medida que se prolonga o tempo de jejum e se restringe a ingestão hídrica, começam a haver alterações no equilíbrio hidroeletrolítico(2). Dentre as respostas fisiológicas que ocorrem com vistas ao seu restabelecimento, a sede é uma das mais relevantes, uma vez que atua na gênese e na cessação da busca por ingestão hídrica. A sede resultante das alterações de osmolaridade e ressecamento de cavidade oral é considerada uma das experiências mais desconfortáveis e estressoras para o paciente no período perioperatório(3-5). Pode ser identificada por um efeito que se autocontrola, chamado de valência negativa(2,6), e é acompanhada por atributos desconfortáveis: boca, lábios e garganta secos, língua e saliva grossas, gosto ruim na boca e vontade de beber água(1,7).

Os atributos relativos à boca, lábios e garganta secos aumentam, de forma exponencial, o desconforto gerado pela sede ${ }^{(1,7)}$. A saliva, que tem papel primordial na hidratação da mucosa, apresenta potencial regulador hídrico do corpo. Em situações em que o organismo está privado de água, ocorre a desidratação da mucosa orofaríngea(8), o que leva à ativação dos osmorreceptores, que, por sua vez, desencadeiam a liberação, entre outros, do hormônio antidiurético $(\mathrm{ADH})$, que atua evitando perda hídrica, até que haja reposição de água. Evidências demostram que, paralelamente, esses osmorreceptores, por meio de vias aferentes, ativam os núcleos osmossensitivos da lâmina terminal, que são reconhecidamente responsáveis pelo controle da sede ${ }^{(2,6,9)}$.

Há dois mecanismos de controle da saciedade da sede: o pós-absortivo, em que a ativação da saciedade é mais lenta, pois o líquido deverá ser absorvido até o alcance do balanço hidromineral, e o mecanismo da saciedade pré-absortiva, em que são ativos os termorreceptores e osmorreceptores orofaríngeos e gástricos, que sinalizam precocemente para o cérebro a interrupção da liberação de $\mathrm{ADH}$ e da consequente sensação de $\operatorname{sede}^{(2)}$. Dessa forma, para o paciente cirúrgico, a utilização de estratégias que estimulem a saciedade pré-absortiva é a mais adequada, uma vez que ocorre mesmo com baixos volumes.
O uso de estratégias para aliviar a sede do paciente cirúrgico no pré-operatório não faz parte da cultura das instituições de saúde, que ainda convivem com preconceitos a respeito da administração de qualquer método de alívio da sede no pré-operatório. Na prática clínica, são registrados até mesmo atrasos e suspensões cirúrgicas por parte de anestesiologistas e cirurgiões quando o paciente faz uso de gomas de mascar, devido ao temor do aumento do conteúdo gástrico. No entanto, metanálise recente comprovou que o uso de gomas de mascar não aumenta o volume e a acidez gástricos de forma clinicamente significativa a ponto de desencadear broncoaspiração(10). A goma de mascar age no aumento do $\mathrm{pH}$ e fluxo salivar por meio de uma combinação da estimulação gustatória e mecânica das glândulas salivares ${ }^{(11)}$, diminuindo a secura da boca e os malefícios que esse sintoma traz.

Adicionalmente, o mentol na goma de mascar atua nos receptores orofaríngeos denominados Transient Receptor Potential Melastatin 8 (TRPM 8), presentes nas terminações nervosas dos nervos trigêmeo $e$ glossofaríngeo, que podem estar relacionados à saciedade em razão de seu trajeto anatômico, com conexões com o hipotálamo e região somatossensorial no córtex ${ }^{(2,9,12)}$.

Estudos com alto nível de evidência têm avaliado o uso da goma de mascar em diversos cenários hospitalares visando a saciar a sede pela estimulação da produção salivar(13-15) e apontam seus benefícios para a redução da sede e xerostomia. Entretanto, não se encontram evidências científicas de estudos bem controlados a respeito do uso da goma de mascar mentolada para a diminuição da intensidade e do desconforto da sede no período pré-operatório, apontando, dessa forma, a relevância da pesquisa. Além disso, a abordagem inovadora proporcionará auxílio aos profissionais no manejo da sede, contribuindo com o aumento da qualidade no cuidado.

Em vista disso, o objetivo deste estudo é avaliar a efetividade da goma de mascar mentolada em aliviar a intensidade e o desconforto da sede do paciente cirúrgico no período pré-operatório.

\section{Método}

Ensaio clínico controlado randomizado, com tratamentos em paralelo, constituído por dois grupos: grupo-controle (GC), que recebeu cuidado usual, ou seja, nenhuma intervenção para o alívio da sede, e grupo experimental (GE), que recebeu goma de mascar mentolada.

Seguiram-se as recomendações do Consolidated Standard Protocol Itens: Recommendations for 
Interventional Trials (SPIRIT) ${ }^{(16)}$ para o protocolo de pesquisa, que foi submetido ao registro de ensaios clínicos randomizados no clinicaltrials.gov da U.S. National Institutes of Health, obtendo o número NCT03200197. Para a elaboração do desenho de estudo, seguiu-se o Consolidated Standards of Reporting Trials (CONSORT) ${ }^{(17) .}$

Em cumprimento à Resolução no 466/12, do Conselho Nacional de Saúde, a pesquisa foi aprovada pelo Comitê de Ética em Pesquisa Envolvendo Seres Humanos, da Universidade Estadual de Londrina, com parecer 1.770.051 e CAAE 59936316.5.0000.5231.

O estudo foi conduzido nas unidades de internação de um hospital universitário de nível terciário no Estado do Paraná. Trata-se de uma instituição pública, com 316 leitos, do Sistema Único de Saúde (SUS), que realiza uma média mensal de 640 cirurgias eletivas e de urgência.

A amostra do estudo foi constituída de pacientes de ambos os sexos internados no hospital selecionado, submetidos à cirurgia eletiva e que atenderam aos critérios de inclusão.

Os critérios de inclusão foram: cirurgia eletiva; idade entre 12 e 65 anos; não ter recebido medicação pré-anestésica; orientado no tempo e espaço; apresentar dentição (natural ou artificial); jejum mínimo de três horas; estar disponível para a coleta, no mínimo, três horas antes do procedimento cirúrgico; verbalizar sede espontaneamente ou, quando questionado, com intensidade maior ou igual a três na Escala Verbal Numérica (EVN) ${ }^{(18)}$.

Para esta avaliação, o paciente deveria responder a cinco perguntas do pesquisador: qual é o seu nome?; qual é a sua idade?; qual é a sua cidade de procedência?; que dia é hoje?; estamos no período da manhã ou da tarde?

Os critérios de exclusão foram: paciente com alergia ao mentol; restrição à mastigação e/ou deglutição; presença de náusea, vômito ou dor no momento da abordagem; xerostomia crônica; paciente renal crônico; impossibilidade de comunicação.

O desfecho clínico primário de interesse foi a variação da intensidade da sede, avaliada pela EVN(18), que varia de zero (sem sede) a dez (sede intensa). 0 desfecho clínico secundário foi a variação do desconforto da sede, avaliada pela Escala de Desconforto da Sede Perioperatória (EDESP), que varia de zero (nenhum desconforto) a 14 (muito desconfortável) e apresenta sete atributos: boca seca, lábios ressecados, língua grossa, saliva grossa, garganta seca, gosto ruim na boca e vontade de beber água(7). A EDESP foi elaborada e validada para mensurar o desconforto causado pela sede no paciente cirúrgico, apresenta índice de conteúdo de 0,98 e índice de fidedignidade de um, consistência interna avaliada pelo alfa de Cronbach de 0,91 e equivalência interobservadores de um medida pelo coeficiente de Kappa ponderado(7). A variável de tratamento do estudo foi a utilização da goma de mascar mentolada, oferecida ao paciente, no mínimo, três horas antes do procedimento anestésico-cirúrgico.

A randomização do teste-piloto e a randomização do estudo foram realizadas por intermédio de lista gerada pelo programa Microsoft Office Exce ${ }^{\circledR}$, com participantes distribuídos aleatoriamente em oito blocos com número diferente de participantes em cada, compondo, desse modo, o GC (cuidado usual) e o GE (goma de mascar mentolada).

A ocultação da alocação foi feita utilizando-se envelopes individuais opacos, numerados externamente de forma sequencial, contendo, em seu interior, a informação do grupo definido pela alocação randômica. O procedimento foi realizado por um profissional que não mantinha contato com a pesquisadora principal. A fim de garantir o cegamento da alocação dos participantes até o momento da intervenção, os envelopes só foram abertos após a aplicação inicial das escalas EVN e EDESP.

Para a coleta dos dados, utilizaram-se três instrumentos: um roteiro e as escalas EVN e EDESP. O roteiro foi submetido à validação aparente por cinco juízes, especialistas em Enfermagem perioperatória e componentes do Grupo de Estudo e Pesquisa da Sede (GPS), com questões demográficas (sexo e idade) e clínicas (clínica cirúrgica, tempo de jejum de sólidos, tempo de jejum de líquidos, índice American Society of Anesthesiologist (ASA), utilização de opioides e anticolinérgicos).

Em razão da inexistência de estudos semelhantes, realizou-se um teste-piloto com 40 pacientes, divididos em dois grupos de 20, que constituíram o GC e o GE. Com período de coleta de dados de novembro a dezembro de 2016, o teste-piloto seguiu todas as etapas metodológicas do ensaio clínico, e seus integrantes não compuseram a amostra final da pesquisa.

O teste-piloto embasou o cálculo amostral, com variação de 1,53 na intensidade da sede. Consideraram-se o nível de significância para o cálculo amostral de 5\%, o intervalo de confiança com nível de $95 \%$ e o poder do estudo de $80 \%$. Os cálculos indicaram uma amostra necessária de 88 pacientes, sendo acrescentados $15 \%$ deste total para casos de perdas de participantes, perfazendo um total de 102 pacientes (51 por grupo) ${ }^{(19)}$.

A goma de mascar de escolha para o testepiloto foi a VALDA $X^{\circledR}$, disponível comercialmente, e o tempo de intervenção estabelecido foi de 20 minutos. Verificou-se, porém, a dificuldade de alguns pacientes degustarem a goma durante todo esse período. Com 
os últimos cinco participantes que utilizaram o produto durante o teste-piloto, observou-se mudança de textura do produto na boca dos pacientes, representando um possível risco de deglutição de pequenos pedaços da goma. Por esse fato, foi necessária a mudança do produto para a goma TRIDENT ${ }^{\circledR}$ de menta, também disponível comercialmente, com composição e gramatura similares às do produto utilizado no teste-piloto, mas de consistência mais firme.

O tempo de intervenção para o estudo foi reduzido de vinte para dez minutos, diante da dificuldade encontrada na degustação durante o teste-piloto. Ao longo do período de intervenção, a pesquisadora permaneceu junto do participante, tanto no GC quanto no GE. Não houve alteração no roteiro de coleta de dados.

O período da coleta de dados do estudo deu-se de janeiro a março de 2017, de acordo com a sequência de procedimentos:

1. No pré-operatório, todos os pacientes que atenderam aos critérios de elegibilidade foram convidados a participar da pesquisa. Os concordantes adultos assinaram o Termo de Consentimento Livre e Esclarecido (TCLE); já os participantes menores de idade assinaram o Termo de Assentimento e seus responsáveis, o TCLE;

2. Coleta de dados demográficos e clínicos nos prontuários;

3. Avaliação inicial da intensidade da sede pela EVN e do desconforto da sede pela EDESP;

4. Alocação randômica e oculta, compondo os grupos GE e GC;

5. Administração da intervenção referente ao grupo alocado, sendo que, no $G E$, cada um recebeu uma unidade de goma de mascar mentolada TRIDENT ${ }^{\circledR}$, mastigando e deglutindo a saliva em ritmo natural durante dez minutos e, no GC, cada um recebeu o cuidado usual realizado nas unidades de internação da instituição em estudo, ou seja, não se fez nenhuma intervenção durante dez minutos de acompanhamento;

6. Avaliação final da intensidade da sede pela EVN e do desconforto da sede pela EDESP, após os dez minutos de intervenção, para os dois grupos.

No GC, pelo fato de os pacientes apresentarem sede intensa, também Ihes era oferecido, após o término da avaliação da coleta, uma goma de mascar mentolada para que aliviassem sua sede.

O procedimento de análise estatística foi mascarado, uma vez que, antes de os dados serem disponibilizados, o GC foi codificado em G1 e o GE, em G2, para impedir que o estatístico distinguisse o grupo que recebeu a intervenção.
Para a análise dos dados, foram utilizados testes não paramétricos, em razão da distribuição anormal da amostra evidenciada pelo Teste de Shapiro-Wilk. As variáveis intensidade e desconforto da sede foram consideradas quantitativas discretas ${ }^{(20)}$.

O Teste de Mann-Whitney foi utilizado para comparar a intensidade e o desconforto da sede inicial, final e a variação entre os dois grupos(20). Para todas as comparações, adotou-se um nível de significância de $5 \%$, com intervalo de confiança de $95 \%$.

Para analisar a correlação entre as variações da intensidade e do desconforto da sede e o uso da goma de mascar, foi aplicado o Coeficiente de Correlação de Spearman ( $\rho)$, com o intervalo de confiança (IC) de $95 \%{ }^{(20)}$. A força da análise foi baseada no tamanho de efeito do d de Cohen: pequeno $(0,20-0,49)$, médio $(0,50-0,79)$ ou grande $(0,80-1,29)^{(21)}$. As análises foram realizadas por meio do programa IBM - SPSS ${ }^{\circledR}$ (versão 20.0).

\section{Resultados}

No período de estudo, 762 pacientes compuseram as listas cirúrgicas eletivas. Desses, 547 estavam fora dos critérios de elegibilidade (idade; estar disponível para a coleta, no mínimo, três horas antes do procedimento cirúrgico; apresentar condições clínicas). Os 215 pacientes remanescentes foram avaliados quanto aos demais critérios de elegibilidade (orientado no tempo e espaço; apresentar dentição; jejum mínimo de três horas; apresentar sede com intensidade maior ou igual a três pela EVN). Os pacientes elegíveis foram convidados a participar do estudo, perfazendo, assim, uma amostra final de 51 pacientes por grupo, aleatorizados para o GC e GE. Não houve perda de segmentos dos participantes (Figura 1).

Constatou-se que não havia diferença estatisticamente significante entre os grupos em relação às variáveis demográficas e clínicas antes da aleatorização (Tabela 1). O teste de normalidade utilizado foi o Shapiro-Wilk e este não apresentou simetria de distribuição. Em vista disso, os testes estatísticos utilizados foram os não paramétricos.

Ao considerar a variação da intensidade da sede, - GE apresentou melhora significativa (mediana $=3$ ) quando comparado ao GC (mediana $=0)(<0,001)$, e o d de Cohen obteve efeito médio $(0,77)^{(21)}$ (Tabela 2). Encontrou-se resultado similar ao observado na variação do desconforto, com o GE obtendo variação ( mediana $=5)$ e o GC, sem (mediana $=0)(p<0,001)$, com d de Cohen com efeito grande $(0,82)^{(21)}$ (Tabela 2 ).

$\mathrm{Na}$ avaliação do desconforto inicial, observou-se alta porcentagem de pacientes com este sintoma nos dois grupos. Já no momento final de avaliação, o GE apresentou melhora, ou seja, diminuição nos valores iniciais em todos os atributos avaliados pela EDESP (Tabela 3). 


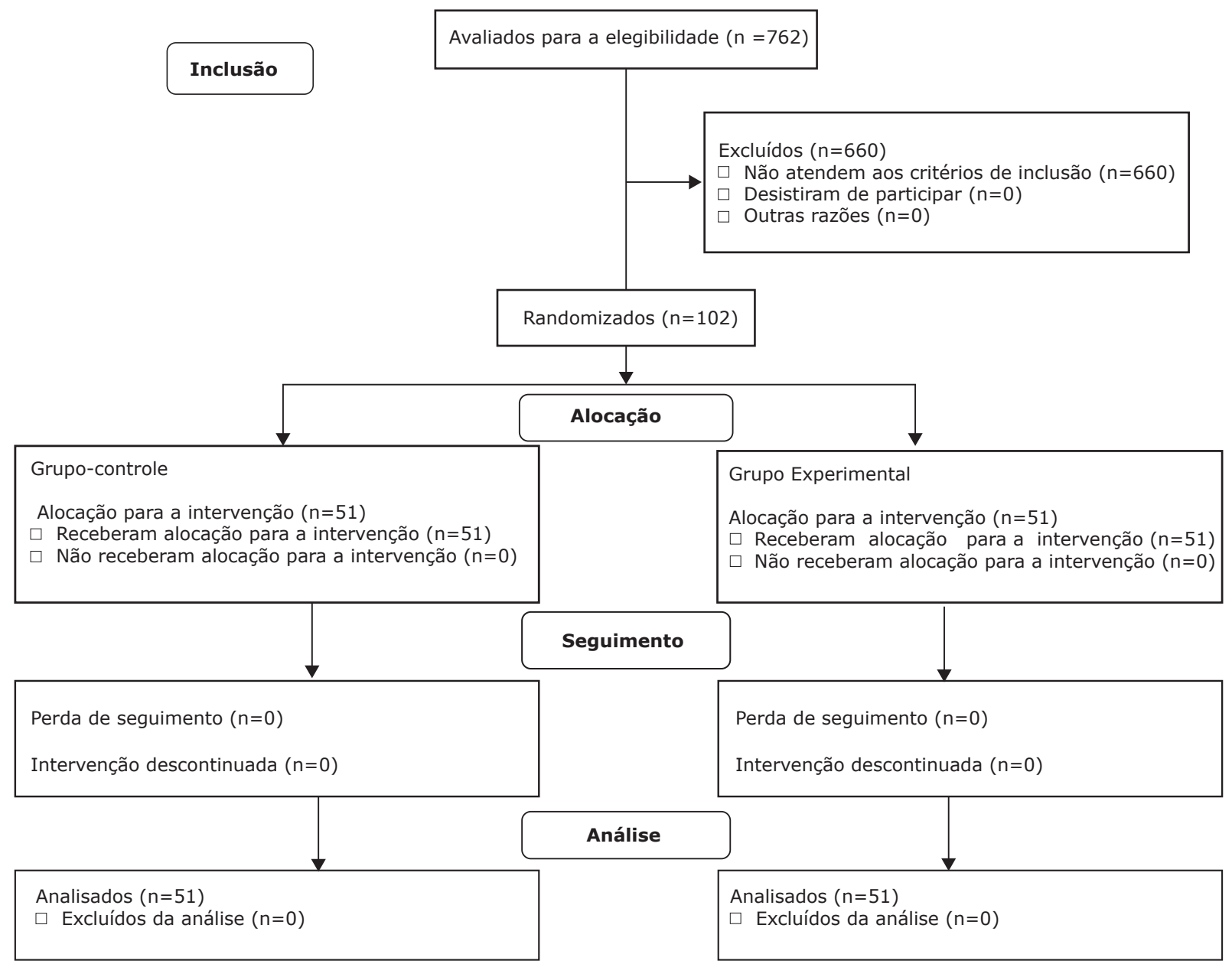

Figura 1 - Diagrama Consort de amostragem e aleatorização. Londrina, PR, Brasil, 2017

Tabela 1 - Distribuição de características demográficas e clínicas segundo os grupos controle e experimental. Londrina, PR, Brasil, 2017

\begin{tabular}{|c|c|c|c|}
\hline Variáveis & $\begin{array}{c}\text { Grupo-controle }(n=51) \\
\text { mediana } \\
\left( \pm 1^{\circ}-3^{\circ} \text { quartil }\right)\end{array}$ & $\begin{array}{c}\text { Grupo experimental }(n=51) \\
\text { mediana } \\
\left( \pm 1^{\circ}-3^{\circ} \text { quartil }\right)\end{array}$ & P valor* \\
\hline Idade (anos) & $43,5(31-49,2)$ & $34,0(23-41,2)$ & 0,124 \\
\hline Jejum sólido $(h)^{\dagger}$ & $11,94(10,5-13,2)$ & $12,75(10,6-15,0)$ & 0,425 \\
\hline \multirow[t]{2}{*}{ Jejum líquido (h) } & $10,97(9,4-12,1)$ & $11,08(10,0-14,2)$ & 0,279 \\
\hline & n (\%) & n (\%) & $P$ valor* \\
\hline \multicolumn{4}{|l|}{ Sexo } \\
\hline Feminino & $30(58,8)$ & $29(56,9)$ & 0,842 \\
\hline Masculino & $21(41,2)$ & $22(43,1)$ & \\
\hline \multicolumn{4}{|l|}{$\mathrm{ASA}^{\ddagger}$} \\
\hline 1 & $29(56,9)$ & $36(70,6)$ & 0,117 \\
\hline II & $18(35,3)$ & $14(27,5)$ & \\
\hline III & $4(7,8)$ & $1(2,0)$ & \\
\hline \multicolumn{4}{|l|}{ Opioides } \\
\hline Sim & $16(31,4)$ & $11(21,6)$ & 0,264 \\
\hline Não & $35(68,6)$ & $40(78,4)$ & \\
\hline
\end{tabular}

${ }^{* P}$ valor $=$ Teste de Mann-Whitney; ${ }^{\text {th }}=$ horas; ${ }^{*}$ ASA $=$ American Society of Anesthesiologist 
Tabela 2 - Comparação entre os grupos controle e experimental em relação à intensidade e desconforto da sede. Londrina, PR, Brasil, 2017

\begin{tabular}{|c|c|c|c|c|}
\hline Desfechos & $\begin{array}{c}\text { Grupo-controle } \\
(n=51) \\
\text { mediana } \\
\left( \pm 1^{\circ}-3^{\circ} \text { quartil }\right)\end{array}$ & $\begin{array}{c}\text { Grupo } \\
\text { experimental } \\
(n=51) \\
\text { mediana } \\
\left( \pm 1^{\circ}-3^{\circ} \text { quartil }\right)\end{array}$ & P valor* & $d_{z}^{\dagger}$ \\
\hline Intensidade inicial & $5,0(4,0-7,0)$ & $6,0(5,0-6,7)$ & 0,68 & - \\
\hline Intensidade final & $5,0(4,0-7,0)$ & $3,0(2,0-4,0)$ & $<0,001$ & 0,60 \\
\hline $\begin{array}{l}\text { Variação da } \\
\text { intensidade }\end{array}$ & $0,0(0,0-0,0)$ & $3,0(1,2-4,7)$ & $<0,001$ & 0,77 \\
\hline Desconforto inicial & $8,5(3,75-12,0)$ & $6,5(3,0-10,7)$ & 0,59 & - \\
\hline Desconforto final & $9,5(3,5-12,7)$ & $1,0(1,0-2,0)$ & $<0,001$ & 0,79 \\
\hline $\begin{array}{l}\text { Variação do } \\
\text { desconforto }\end{array}$ & $0,0(-0,7-0,0)$ & $5,0(1,2-8,0)$ & $<0,001$ & 0,82 \\
\hline
\end{tabular}

*P valor = Teste de Mann-Whitney; ${ }^{+} \mathrm{d}_{\mathrm{z}}=\mathrm{d}$ de Cohen extraído do valor $\mathrm{Z}$

Tabela 3 - Frequência dos atributos da Escala de Desconforto da Sede Perioperatória antes e depois da intervenção nos grupos controle e experimental. Londrina, PR, Brasil, 2017

\begin{tabular}{lcccc}
\hline \multirow{2}{*}{ Atributos da EDESP* } & \multicolumn{3}{c}{ Grupo-controle } & \multicolumn{2}{c}{ Grupo experimental } \\
\cline { 2 - 5 } & $\begin{array}{c}\text { Antes } \\
\%(\mathbf{N})\end{array}$ & $\begin{array}{c}\text { Depois } \\
\%(\mathbf{N})\end{array}$ & $\begin{array}{c}\text { Antes } \\
\%(\mathbf{N})\end{array}$ & $\begin{array}{c}\text { Depois } \\
\%(\mathbf{N})\end{array}$ \\
\hline Boca seca & $64,7(33)$ & $72,5(37)$ & $64,7(33)$ & $3,9(2)$ \\
Lábios ressecados & $60,8(31)$ & $62,7(32)$ & $58,8(30)$ & $15,7(8)$ \\
Língua grossa & $41,2(21)$ & $39,2(20)$ & $49,0(25)$ & $11,8(6)$ \\
Saliva grossa & $62,7(32)$ & $62,7(32)$ & $47,1(24)$ & $2,0(1)$ \\
Garganta seca & $56,9(29)$ & $60,8(31)$ & $62,7(32)$ & $3,9(2)$ \\
Gosto ruim na boca & $58,8(30)$ & $60,8(31)$ & $49,0(25)$ & $0,0(0)$ \\
Vontade de beber água & $100,0(51)$ & $98,0(50)$ & $100,0(51)$ & $66,7(34)$ \\
\hline *EDESP = Escala de Desconforto da Sede Perioperatória
\end{tabular}

A Correlação de Spearman evidenciou que as variações da intensidade e do desconforto têm relação positiva e forte ( $\rho=0,841, p<0,0001)$ e tiveram relação com o uso da goma de mascar ( $\rho=0,778$ e 0,831 $\mathrm{p}<0,0001$ ) (Figura 2).

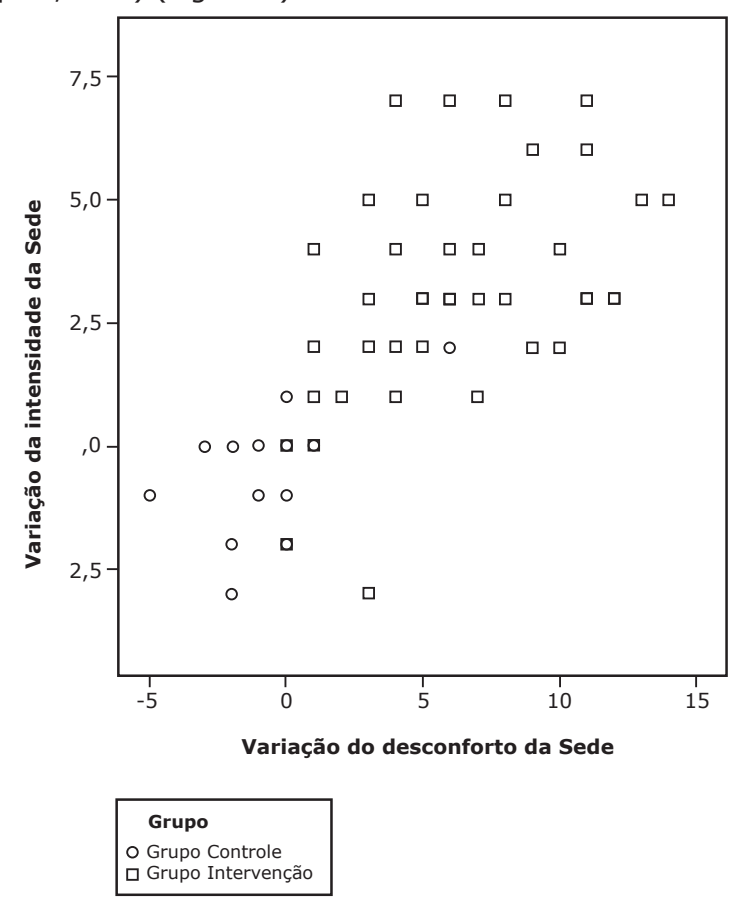

Figura 2 - Gráfico de dispersão do Coeficiente de Correlação de Spearman sobre a variação da intensidade e do desconforto da sede entre os grupos. Londrina, PR, Brasil, 2017

\section{Discussão}

Este estudo apresentou uma abordagem inovadora ao avaliar uma estratégia simples, viável, prática, de baixo custo, efetiva e fundamentada em mecanismos fisiológicos que atuam minimizando a sede e seus desconfortos. Além disso, apresenta evidências sustentadas que contrapõem o paradigma cultural de que não se pode intervir na sede pré-operatória. Somado a isso, tanto no teste-piloto quanto no estudo final não houve eventos adversos relativos à administração da goma de mascar.

Não obstante, a equipe segue com a conduta de reforçar insistentemente a impossibilidade de ingestão de qualquer quantidade de líquidos ${ }^{(22-23)}$. Dentre as contribuições desta pesquisa, está a constatação de que o paciente no pré-operatório também sente sede. Além disso, tanto o grupo experimental quanto o controle apresentaram desconforto acentuado em relação à sede no primeiro momento de avaliação. A goma de mascar mentolada mostrou-se efetiva para o alívio da sede no pré-operatório, considerando os efeitos médio a grande encontrados na variação da intensidade (d de Cohen 0,77 ) e do desconforto ( $d$ de Cohen 0,82 ) da sede após o uso de uma única unidade da goma, pelo período de apenas dez minutos. Já os pacientes que não receberam a intervenção não apresentaram redução da sede.

Estes dados corroboram estudos em que se obteve resultado semelhante em relação à intensidade da sede com o uso da goma de mascar, ainda que realizados com outras populações(13-15). Tais estudos apontam o uso desta estratégia em pacientes com xerostomia, em tratamento dialítico, igualmente submetidos à restrição hídrica(13-14). Além disso, a goma de mascar também foi testada em pacientes com câncer avançado de cabeça e pescoço que, submetidos a sessões de radioterapia, apresentam disfunções de secreção salivar, que leva ao ressecamento da orofaringe e, portanto, à sede ${ }^{(15)}$. O uso da estratégia apresentou efeito positivo na estimulação das glândulas salivares e consequente aumento do fluxo salivar, minorando a sede ${ }^{(13-15)}$.

Os atributos desconfortáveis da sede são relacionados à diminuição salivar e desidratação oral, mostrando-se intensos no período pré-operatório(1-3,7). Neste estudo, evidenciou-se a efetividade da goma de mascar mentolada sobre os desconfortos avaliados pela EDESP. Todos os atributos apresentaram redução significativa após os pacientes receberem uma goma mentolada para mascar por apenas dez minutos.

Demonstrou-se a presença de correlação entre as variáveis intensidade e desconforto, assim como o uso da goma de mascar mentolada, pois, quando uma variável foi reduzida pelo uso da estratégia, a outra apresentou o mesmo comportamento. Um estudo com 203 pacientes, 
em que se avaliou a sede na sala de recuperação anestésica utilizando a EDESP, também encontrou correlação entre intensidade e desconforto da sede ${ }^{(24)}$. Isso demostra que, além de se avaliar a intensidade, é importante mensurar também o desconforto relacionado à sede.

Os dados evidenciaram que a intervenção foi efetiva. Esse efeito positivo da goma de mascar mentolada pode ser explicado por três fatores principais: aumento do fluxo salivar por meio da estimulação das glândulas salivares, presença do mentol e do xilitol na composição da goma.

O volume de produção de secreção ácida estomacal em um indivíduo é, comumente, de $0,6 \mathrm{ml} \cdot \mathrm{kg}^{-1} \cdot \mathrm{h}^{-1}$. Entretanto, se o mesmo indivíduo permanecer em jejum prolongado, como ocorre com pacientes cirúrgicos no período pré-operatório, pode apresentar produção

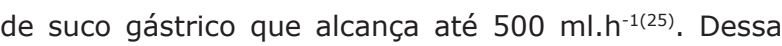
forma, se a taxa de fluxo salivar estimulado pelo uso da goma de mascar é de $6,6 \mathrm{ml}$. $\mathrm{min}^{-1}$ no primeiro minuto de mastigação, diminuindo para $1,5 \mathrm{ml} \cdot \mathrm{min}^{-1}$ dentro de 15 minutos $^{(26-28)}$, o uso da goma de mascar representa fator de proteção para o aumento do conteúdo gástrico.

Sugere-se, ainda, que sentimentos incidentes no período pré-operatório, tais como medo, insegurança e ansiedade, podem gerar estresse cirúrgico, ressecamento da cavidade oral, náusea e hipoglicemia, que estimulam a secreção de $A D H$ e, por conseguinte, a sensação de sede(5). Em um estudo, observou-se que a goma de mascar pode diminuir tanto a ansiedade dos pacientes quanto aumentar o $\mathrm{pH}$ salivar ${ }^{(29)}$. Além disso, a umidificação oral proporcionada por ela e o aumento da deglutição pelo fluxo salivar fazem com que diminua a secreção de $A D H^{(9)}$.

Pesquisas apontam que há preferência por estratégias aromatizadas, quando comparadas à parafina ou à goma de mascar isenta de sabor ${ }^{(13-15)}$. Diversos estudos utilizaram gomas aromatizadas com mentol visando à agradabilidade para o indivíduo por causa do sabor, não por sua particularidade de ativar os receptores TRPM 8, que possuem relação com as vias neurais da sede $\mathrm{e}^{(13-15,29)}$.

Uma limitação do estudo foi o desconhecimento do tipo de mentol que compõe a goma de mascar utilizada pelo fato de a goma escolhida ser comercialmente disponível e sua formulação não ser de acesso público. Além disso, não foi possível avaliar a duração do efeito da estratégia mentolada na intensidade e no desconforto da sede.

Outro fator para a superioridade da intervenção é a presença do edulcorante denominado xilitol, que substitui a sacarose na composição da goma(30). Entre seus benefícios, estão a possibilidade de utilização por diabéticos ${ }^{(30)}$ e seu valor negativo de calor de dissolução $\left(-34,8 \mathrm{cal} . \mathrm{g}^{-1}\right)$, produzindo agradável efeito refrescante na boca ao entrar em contato com a saliva. Devido a essa propriedade organoléptica, o xilitol realça o efeito refrescante ${ }^{(30)}$ dos produtos mentolados como, neste caso, a goma de mascar.

A efetividade da goma de mascar mentolada, em proporcionar a redução da intensidade e do desconforto da sede, pode ser explicada fisiologicamente, uma vez que, durante a mastigação da goma, o mentol mimetiza a ação da temperatura fria e ativa os receptores TRPM8, que decodificam a presença de mentol em impulsos nervosos e os transmitem por meio das fibras sensoriais aferentes aos nervos trigêmeo e glossofaríngeo. Esses nervos têm suas ramificações na cavidade oral, mandíbula e orofaringe, e suas raízes localizam-se no núcleo trigeminal medular e no núcleo do trato solitário, respectivamente, irradiando-se para os núcleos supraóptico, paraventricular e órgão subfornical, que são áreas altamente relacionadas com estímulos de sede e secreção de $\operatorname{ADH}^{(2,7,9,31-32)}$. Ocorre, ainda, a irradiação dessas inervações para o córtex cingulado anterior, mais precisamente para as áreas três, dois e um de Brodmann, também chamado de somatossensorial, que permite a experimentação de sensações distintas, entre elas, a sede e sua saciedade ${ }^{(33-35)}$.

À vista disso, esta estratégia apresenta alta relevância clínica, pois sua utilização é simples e factível no período pré-operatório. Além de se mostrar efetiva, concretiza-se como um desafio ao paradigma estabelecido na prática clínica a respeito de suspensão cirúrgica em caso de uso da goma de mascar por escolha do próprio paciente ${ }^{(10)}$. Além do mais, é de fácil aplicabilidade clínica e representa um aumento na qualidade do cuidado e da humanização, devido ao olhar intencional para uma necessidade básica do ser humano. Acrescenta-se que esta intervenção não farmacológica é de baixo custo e excelente aceitabilidade pelos pacientes ${ }^{(36)}$, que referiram sensação agradável e de intenso conforto com o uso da goma.

\section{Conclusão}

Houve diferenças estatística e clínica significativas em relação à efetividade da estratégia goma de mascar mentolada para o alívio da intensidade e desconforto da sede no paciente cirúrgico em período préoperatório. Perante os resultados evidenciados neste estudo, conclui-se que esta evidência é uma estratégia simples, de alta factibilidade clínica, baixo custo e boa aceitabilidade pelo paciente. Apresenta-se como uma inovação na quebra de um paradigma de que a goma de mascar não pode ser oferecida ao paciente cirúrgico. Também contribui para a ampliação do conhecimento no manejo da sede do paciente cirúrgico, particularmente 
no pré-operatório. Representa valorização do cuidado de Enfermagem de maneira individualizada, uma vez que atende a uma necessidade humana básica tão comumente negligenciada.

\section{Referências}

1. Gebremedhn EG, Nagaratnam VB. Audit on perioperative fasting of elective surgical patients in an African academic medical center. WId J Surg. 2014; 38(9):2200-4. doi: 10.1007/s00268-014-2582-3

2. Zimmerman CA, Leib DE, Knight ZA. Neural circuits underlying thirst and fluid homeostasis. Nat Rev Neurosci. 2017 Aug;18(8):459-69. doi:10.1038/ nrn.2017.71

3. Silva LCJR, Aroni P, Fonseca LF. I am thirsty! Experience of the surgical patient in the perioperative period. Rev SOBECC. 2016; 21(2):75-81. doi: https:// doi.org/10.5327/Z1414-4425201600020003

4. Dessotte CAM, Rodrigues HF, Furuya RK, Rossi LA, Dantas RAS. Stressors perceived by patients in the immediate postoperative of cardiac surgery. Rev Bras Enferm. 2016; 69(4):694-703. doi: http://dx.doi. org/10.1590/0034-7167.2016690418i

5. Conchon MF, Nascimento LA, Fonseca LF, Aroni P. Perioperative thirst: an analysis from the perspective of the Symptom Management Theory. Rev Esc Enferm USP. 2015; 49(1):122-8. doi: 10.1590/S0080-623420150000100016 6. Zimmerman CA, Lin Y, Leib DE, Guo L, Huey EL, Daly GE, et al. Thirst neurons anticipate the homeostatic consequences of eating and drinking. Nature. 2016;537:680-4. doi:10.1038/nature 18950

7. Martins PR, Fonseca LF, Rossetto EG, Mai LD. Developing and validating the Perioperative Thirst Discomfort Scale. Rev Esc Enferm USP. 2017; 51,e03240. doi: http:// dx.doi.org/10.1590/S1980-220X2016029003240

8. Bichet DG. Regulation of Thirst and Vasopressin Release. Annu Rev Physiol. 2019; 10(81):359-73. doi: 10.1146/annurev-physiol-020518-114556.

9. Verbalis JG. Disorders of body water homeostasis. Best Practice \& Research Clinical Endocrinology \& Metabolism. 2003; 17(4):471-503. doi:10.1016/S1521690X(03)00049-6

10. Quanes JP, Bicket MC, Togioka B, Tomas VG, Wu CL, Murphy JM. The role of perioperative chewing gum on gastric fluid volume and gastric $\mathrm{pH}$ : a meta-analysis. J Clin Anesth. 2015; 27(2):146-52. doi: 10.1016/j. jclinane.2014.07.005

11. Oyakawa EHR, Contreras SJS. Tasa de flujo salival y nivel de confort al emplear saliva artificial y caramelos de menta sin azúcar en adultos mayores con xerostomía. Rev Estomatol Herediana. 2006; 16(2):103-9. doi: https://doi.org/10.20453/reh.v16i2.1912
12. Saker P, Farrell MJ, Adib FRM, Egan GF, McKinley MJ, Denton DA. Regional brain responses associated with drinking water during thirst and after its satiation. Proc Natl Acad Sci. 2014; 111(14):5379-84. doi: https://doi. org/10.1073/pnas.1403382111

13. Fan $W F$, Zhang Q, Luo LH, Gu Y. Study on the clinical significance and related factors of thirst and xerostomia in maintenance hemodialysis patients. Kidney Blood Press Res. 2013; 37(4-5):464-74. doi: 10.1159/000355717 14. Bots CP, Brand HS, Veerman ECI, Korevaar JC, Valentijn-Benz $M$, Bezemer PD, et al. Chewing gum and a saliva substitute alleviate thirst and xerostomia in patients on haemodialysis. Nephrol Dial Transplant. 2005; 20:578-84b. doi: 10.1093/ndt/gfh675

15. Davies AN. A comparison of artificial saliva and chewing gum in the management of xerostomia in patients with advanced cancer. Palliative Med. 2000; 14(3):197-203. doi: 10.1191/026921600672294077

16. Calvert M, Kyte D, Mercieca-Bebber R, Slade A, Chan A-W, King MT, et al. Guidelines for Inclusion of PatientReported Outcomes in Clinical Trial Protocols: The SPIRIT-PRO Extension. Jama 2018; 319(5):483-94. doi: 10.1001/jama.2017.21903.

17. Moher D, Hopewell S, Schulz KF, Montori V, Gøtzsche PC, Devereaux PJ, et al. CONSORT 2010 Explanation and Elaboration: Updated guidelines for reporting parallel group randomised trials. BMJ. 2011;343:d6131. doi: https://doi.org/10.1136/bmj.d6131

18. Gries K, Berry P, Harrington M, Crescioni M, Patel $M$, Rudell $K$, et al. Literature review to assemble the evidence for response scales used in patient reported outcome measures. J Patient-Reported Outcome 2018; 2:41. doi: 10.1186/s41687-018-0056-3

19. Miot HA. Sample size in clinical and experimental trials. ] Vasc Bras. [Internet]. 2011 [cited Jun 12, 2018]; 10(4):275-8 Available from: http://www.scielo. $\mathrm{br} / \mathrm{pdf} / \mathrm{jvb} / \mathrm{v} 10 \mathrm{n} 4 / \mathrm{en} \_v 10 \mathrm{n} 4 \mathrm{a01.pdf}$

20. Coutinho ESF, Cunha GM. Basic concepts in epidemiology and statistics for reading controlled clinical trials. Rev Bras Psiquiatr. 2005; 27:146-51. doi: http://dx.doi.org/10.1590/S1516-44462005000200015 21. Espírito-Santo $H$, Daniel F. Calculating and reporting effect sizes on scientific papers (1): $p<0.05$ limitations in the analysis of mean differences of two groups. Portuguese J Behav Soc Res. 2015; 1(1): 3-16. doi: 10.7342/ismt.rpics.2015.1.1.14

22. Pavani MM, Fonseca LF, Conchon MF. Thirst in surgical patients: perceptions of the nursing team in inpatient units. Rev enferm UFPE on line. 2016; 10(9):3352-60. doi: 10.5205/reuol.9571-83638-1-SM1009201621

23. Garcia ACKA, Nascimento LA, Conchon MF, Garcia AKA, Fonseca LF. Anesthesiologist's perspective regarding thirst in the immediate postoperative period. 
Cienc Cuid Saúde 2017. Jul-Set; 16(3). doi: 10.4025/ cienccuidsaude.v16i3.37241

24. Pierotti I, Fracarolli IL, Fonseca LF, Aroni P. Evaluation of the intensity and discomfort of perioperative thirst. Esc Anna Nery. 2018;22(3):e20170375. doi: 10.1590/21779465-EAN-2017-0375

25. Moro TE. Prevention of Pulmonary Gastric Contents Aspiration. Rev Bras Anestesiol. [Internet]. 2004 [cited Jun 12, 2018]; 54(2):261-75. Available from: http:// www.scielo.br/pdf/rba/v54n2/v54n2a14.pdf

26. Dubin SA, Jense HG, McCranie JM, Zubar V. Sugarless gum chewing before surgery does not increase gastric fluid volume or acidity. Can J Anaesth. 1994; 41(7):603-6. doi: 10.1007/BF03010000

27. Dawes C, Macpherson LMD. Effects of nine different chewing-gums and lozenges on salivary flow rate and $\mathrm{pH}$. Caries Res. 1992; 26(3):176-82. doi: 10.1159/000261439

28. Søreide $E$, Holst-Larsen $H$, Veel T, Steen PA. The effects of chewing gum on gastric content prior to induction of general anesthesia. Anesth Analg [Internet]. 1995 [cited Jun 12, 2018]; 80(5):985-9. Available from: https://www.ncbi.nlm.nih.gov/pubmed/7726444

29. Hamid K, Masoud L, Reza FH, Mehran G, Karmella K. Comparison of different non-pharmacological preoperative preparations on gastric fluid volume and acidity: a randomized controlled trial. Anaesth Pain Intensive Care [Internet]. 2012 [cited Jun 12, 2018];16:165-8. Available from: goo.gl/4sMTTx

30. Maia MCA, Galvão APGLK, Modesta RCD, Pereira Junior N. Consumer evaluation of ice cream with xylitol. Ciênc Tecnol Aliment. [Internet]. 2008 [cited Jun 12, 2018];28(2):341-47. Available from: http://www.scielo. $\mathrm{br} / \mathrm{pdf} / \mathrm{cta} / \mathrm{v} 28 \mathrm{n} 2 / \mathrm{a} 11 \mathrm{v} 28 \mathrm{n} 2 . \mathrm{pdf}$

31. Salata RA, Verbalis JG, Robinson AG. Cold water stimulation of oropharyngeal receptors in man inhibits release of vasopressin. J Clin Endocrinol Metab. 1987; 65(3):561-7. doi: 10.1210/jcem-65-3-561

32. Gizowski C, Bourque CW. Neurons that drive and quench thirst. Median preoptic neurons modulate water intake and the urge to drink. Science. 2017; 357(6356):1092-3. doi: 10.1126/science.aao5574.

33. Peier AM, Mogrich A, Hergarden AC, Reeve AJ, Anderson DA, Story GM, et al. A TRP channel that senses cold stimuli and menthol. Cell. [Internet]. 2002 [cited Jun 12, 2018]; 108(5):705-715. Available from: https://www.ncbi.nlm.nih.gov/pubmed/11893340

34. Latorre R, Brauchi S, Madrid R, Orio P. A cool channel in cold transduction. Physiology (Bethesda). 2011; 26(4):273-85. doi: 10.1152/physiol.00004.2011 35. Eccles R, Du-Plessis L, Dommels Y, Wilkinson JE. Cold pleasure: why we like ice drinks, ice-lollies and ice cream. Appetite. 2013; 71:357-60. doi: 10.1016/j. appet.2013.09.011

36. Olsson $\mathrm{H}$, Spak CJ, Axéll T. The effect of a chewing gum on salivary secretion, oral mucosal friction, and the feeling of dry mouth in xerostomic patients. Acta Odontol Scand. [Internet]. 1991 [cited Jun 12, 2018]; 49(5):273-9. Available from: https://www.ncbi.nlm.nih. gov/pubmed/1803848
Recebido: 01.11.2018

Aceito: 15.04 .2019
Copyright $\odot 2019$ Revista Latino-Americana de Enfermagem Este é um artigo de acesso aberto distribuído sob os termos da Licença Creative Commons CC BY.

Esta licença permite que outros distribuam, remixem, adaptem e criem a partir do seu trabalho, mesmo para fins comerciais, desde que Ihe atribuam o devido crédito pela criação original. É a licença mais flexível de todas as licenças disponíveis. É recomendada para maximizar a disseminação e uso dos materiais licenciados. 